\title{
Apomorphine, systèmes dopaminergiques centraux et contrôle de l'érection
}

\author{
Olivier RAMPIN \\ Institut National de la Recherche Agronomique, Laboratoire de Neurobiologie des Fonctions Végétatives- \\ Jouy en Josas
}

\section{RÉSUMÉ}

L'érection est un mécanisme vasculotissulaire provoqué par la relaxation des fibres musculaires lisses du pénis et des artères qui l'irriguent. L'érection est contrôlée par le système nerveux autonome, les voies parasympathiques étant proérectiles et les voies sympathiques antiérectiles.

$\mathrm{Au}$ cours des dernières années, les nombreuses données fondamentales élucidant les mécanismes locaux de l'érection ont permis de proposer des traitements des dysfonctions érectiles par des médicaments à action périphérique. Ces produits permettent soit de provoquer une érection, ils sont alors efficaces seuls, soit de potentialiser une érection.

Dans un environnement sexuellement stimulant, l'érection est provoquée par une modification de l'équilibre des effets antiérectiles du système sympathique et des effets proérectiles du système parasympathique. Les voies sympathiques et parasympathiques naissent de la moelle épinière. Le système nerveux central agit sur la moelle épinière pour contrôler et modifier cet équilibre sympathiqueparasympathique. La dopamine est un neuromédiateur du système nerveux central agissant sur le comportement sexuel et sur les noyaux centaux contrôlant le tractus génital chez le mâle. Les taux de dopamine augmentent dans plusieurs noyaux centraux (amygdale, aire préoptique médiane) pendant l'activité sexuelle chez le rat. Les agonistes dopaminergiques à action centrale comme l'apomorphine (agoniste D1/D2) provoquent l'érection chez l'homme, le singe et le rat. Chez le rat, les effets proérectiles de l'apomorphine sont dus à sa fixation sur des récepteurs de type D2 dans le noyau paraventriculaire de l'hypothalamus (PVN), et seraient régulés par la testostérone. Des investi- gations cliniques récentes ont mis en avant les avantages de l'utilisation de l'apomorphine pour traiter les patients souffrant de dysfonctions érectiles. Une molécule à action centrale comme l'apomorphine pourrait réorganiser l'équilibre sympathique-parasympathique, conduisant à un recrutement adapté des voies nerveuses vers le pénis.

Mots-clés : apomorphine, dopamine, érection, éjaculation, dysfonction érectile, comportement sexuel.

\section{L'ÉRECTION}

Les mécanismes locaux de l'érection sont la relaxation des fibres musculaires lisses du pénis et des artères qui l'irriguent. Cette relaxation permet une augmentation du débit sanguin vers le pénis, et le remplissage des espaces caverneux et spongieux par le sang [3]. L'érection est entièrement dépendante du système nerveux autonome. Le système parasympathique issu de la moelle épinière sacrée est pro-érectile, le système sympathique issu de la moelle épinière dorsolombaire est anti-érectile. On considère aujourd'hui que l'érection résulte d'une augmentation de l'activité des voies parasympathiques accompagnée d'une baisse de l'activité des voies sympathiques. Les noyaux spinaux à l'origine des voies sympathique et parasympathique sont mis en jeu par des informations d'origine périphérique, par exemple de la région génitale, et intervien-

Correspondance : Dr. Olivier Rampin - Institut National de la Recherche Agronomique. Laboratoire de Neurobiologie des Fonctions Végétatives. Bâtiment 325. F-78352 JOUY-ENJOSAS - Tel 33.1.3465.2503 - Fax 33.1.3465.2505 - Email rampin@jouy.inra.fr 
nent donc dans des mécanismes réflexes (érection, éjaculation). Ces noyaux sont également contrôlés par des informations excitatrices et inhibitrices issues des noyaux du bulbe rachidien, du pont et de l'hypothalamus [85]. On ignore aujourd'hui comment des informations d'origine périphérique et centrale convergent vers la moelle pour recruter les voies pro-érectiles et déprimer l'activité des voies anti-érectiles.

La commande de l'érection par le système nerveux central est complexe, et l'on commence seulement à la comprendre [1]. Le système nerveux central peut contrôler l'érection par des projections directes (exemple des voies hypothalamospinales) ou relayées vers les noyaux spinaux pro-érectiles. Il peut être également permissif vis-à-vis de l'érection, par exemple en contrôlant le comportement sexuel. L'érection apparaît dans différentes situations (érections durant le coït, réflexe, durant le sommeil, érections psychogènes, provoquées pharmacologiquement) et l'on a émis l'hypothèse que des mécanismes nerveux différents intervenaient dans ces différentes situations [114]. Dans le système nerveux central, le système dopaminergique a reçu une attention spéciale et son rôle dans les mécanismes du comportement sexuel et de l'érection a été plus particulièrement étudié. Les voies dopaminergiques centrales comprennent les systèmes nigro-strié, mésolimbique, mésocortical, incertohypothalamique, hypothalamospinal et tubérohypophysaire.

Les systèmes dopaminergiques interviennent dans les mécanismes du comportement sexuel et de la motricité génitale [15, 86, 93]. Les projections dopaminergiques vers le striatum réguleraient la coordination motrice durant le coït, les projections vers le système limbique et le cortex participeraient à la mise en place de l'excitation sexuelle, de la motivation et de la récompense, et les projections vers l'hypothalamus contribueraient à la performance sexuelle et au contrôle des organes génitaux. Dans le cerveau, la dopamine, synthétisée et libérée par les neurones, agit sur les cellules cibles par l'intermédiaire de cinq sous-types de récepteurs, D1-D5. Ces récepteurs ont été caractérisés par les techniques de la biologie moléculaire [96]. On classe ces récepteurs en D1-like (D1 et D5) et D2-like (D2, D3 et D4). Les propriétés pharmacologiques des récepteurs D1like et D2-like sont différentes.

\section{DOPAMINE, APOMORPHINE ET COMPORTEMENT SEXUEL}

Chez le rat mâle, les quantités de dopamine et de son métabolite, l'acide dihydroxyphénylacétique, augmentent dans l'aire préoptique médiane, le noyau accumbens, et l'hypothalamus médiobasal quand le mâle est mis en présence d'une femelle réceptive, et continuent d'augmenter durant le coït $[47,68,107,109]$. L'aire préoptique médiane est une structure clé du contrôle central du comportement sexuel [86]. Selon une hypothèse, l'augmentation des quantités de dopamine centrale dans l'aire préoptique médiane permettrait de recruter successivement plusieurs sous-types de récepteurs dopaminergiques. Ces différents récepteurs contrôleraient des aspects différents du comportement sexuel et de la motricité génitale. On pourrait ainsi expliquer l'évolution des réponses sexuelles de l'excitation à l'éjaculation via l'érection et l'émission [67].

Les effets des agonistes et des antagonistes dopaminergiques ont été étudiés sur le comportement sexuel chez le rat. Chez cette espèce, le mâle réalise une série de montes, accompagnées ou non d'intromission, s'achevant par l'éjaculation. Une monte ou une intromission ne durent pas plus d'une seconde, et l'éjaculation dure seulement quelques secondes. Des rats mâles adultes sexuellement expérimentés réalisent quelques montes et de 5 à 10 intromissions avant l'éjaculation. Cette dernière apparaît environ 10 minutes après la présentation de la femelle. Une période réfractaire durant plusieurs minutes suit l'éjaculation. Le mâle reprend ensuite son activité sexuelle. La latence de la première monte et le nombre de montes reflètent la motivation sexuelle, et la latence de l'intromission, le nombre d'intromissions, la latence de l'éjaculation et le nombre d'éjaculations sont des témoins de la performance sexuelle.

Les produits qui altèrent la transmission dopaminergique centrale (halopéridol, pimozide, chlorpromazine, alphamethyl-tyrosine) affaiblissent ou éliminent le comportement sexuel du rat $[81,126]$. Les produits qui facilitent ou miment la transmission dopaminergique centrale stimulent le comportement sexuel : chez le rongeur, cela se traduit par une diminution de la latence de la première monte ou intromission, l'augmentation relative du nombre d'intromissions par rapport au nombre de montes, la diminution du nombre de montes et d'intromissions avant l'éjaculation, la diminution de la latence de l'éjaculation, la diminution de la période réfractaire, l'augmentation du nombre d'éjaculations durant un test $[82,126,127]$. On compte, parmi ces produits facilitateurs du comportement sexuel, l'amantadine, qui déclenche une libération de dopamine [45, 46], et les agonistes dopaminergiques D2 : RDS-127 (2-N,N-di-n-propylamino-4,7-dimethoxyindane, [23, 24, 25]), et SND 919 (pramipexole, [44]), l'apomorphine, la N-n-propyl-norapomorphine, la 3-(3-hydroxyphenyl )-N-n-propyl-piperidine (3-PPP), le lisuride, la L-DOPA, SND 919 et B-HT 920 [11, $12,50,100,103]$. Chez le singe, l'apomorphine et le quinelorane (LY 163502) déclenchent la masturbation en présence d'une femelle réceptive [110, 111].

Les effets de l'apomorphine délivrée par voie intracérébroventriculaire ou injectée directement dans l'aire préoptique médiane sur le comportement sexuel du rat sont biphasiques : délétères à faible dose, facilitateurs à haute dose 
[37, 65]. L'aire tegmentale ventrale émet des projections dopaminergiques vers le système limbique. L'injection d'apomorphine dans l'aire tegmentale ventrale ralentit la copulation chez le rat [66]. Certains rats mâles présentent spontanément une faible activité sexuelle. Le pourcentage de tels rats présentant une éjaculation dans un test de copulation de trente minutes est faible. Si l'on injecte de l'apomorphine ou de la L-DOPA à ces rats, on augmente le pourcentage d'animaux parvenant à l'éjaculation avant la fin du test $[127,128]$. On sait aujourd'hui "construire" des souris chez lesquelles les gènes codant pour des neuromédiateurs ont été inactivés. Des souris incapables de synthétiser la dopamine ont été ainsi obtenues. Pour survivre, ces animaux reçoivent des injections de L-DOPA. Ce traitement stimule le comportement de monte [125].

L'ensemble des données montre un rôle majeur du système dopaminergique central dans le contrôle du comportement sexuel. L'effet est principalement dû au recrutement de récepteurs dopaminergiques $\mathrm{D} 2$.

\section{DOPAMINE, APOMORPHINE, ÉRECTION ET ÉMISSION SÉMINALE}

Des agonistes dopaminergiques, dont l'apomorphine, déclenchent des érections chez l'animal et chez l'homme. Les voies d'administration sont multiples : injections souscutanée, intramusculaire, intrapéritonale, intraveineuse, prises orale et sublinguale, injections intracérébroventriculaire, intrathécale et dans certains noyaux centraux. Le tableau 1 résume les doses d'apomorphine et ses voies d'injections qui déclenchent des érections chez plusieurs espèces animales et chez l'homme.

Les effets proérectiles de l'apomorphine sont dépendants de la dose utilisée, et la courbe dose-réponse suit une forme en cloche. Chez le rat et le singe, l'apomorphine déclenche des érections en l'absence de tout autre stimulation [11, 48]. Chez le rat éveillé, chaque érection déclenchée par l'apomorphine et identifiée à partir de critères comportemen- taux, est accompagnée d'une augmentation de la pression intracaverneuse [14]. Les doses d'apomorphine déclenchant l'érection chez le rat $(50-100 \mu \mathrm{g} / \mathrm{kg})$ sont proches de celles déclenchant deux autres comportements, le syndrome de 1' "étirement-bâillement" ("stretching-yawning syndrome" des auteurs anglo-saxons), et la toilette du corps ("grooming"). En revanche ces doses sont inférieures aux doses qui provoquent des stéréotypies. On peut donc observer et quantifier une série de comportements déclenchés par l'apomorphine et les produits agissant sur la transmission dopaminergique.

Benassi-Benelli et coll. [11] ont été parmi les premiers à fournir une description précise et complète des effets proérectiles de l'apomorphine chez le rat éveillé. Leur approche pharmacologique a ainsi révélé que l'injection intrapéritonéale d'apomorphine et de N-n-propyl-norapomorphine déclenchait des érections d'une façon dosedépendante. Les effets proérectiles sont abolis après injection d'halopéridol et de sulpiride, antagonistes dopaminergiques à effet central, mais pas par la dompéridone, antagoniste dopaminergique à effet périphérique. Depuis, plusieurs études ont confirmé ces données, et ont montré que d'autres agonistes dopaminergiques, ou des produits mimant ou amplifiant la neurotransmission dopaminergique centrale, possédaient des propriétés proérectiles : l'apocodéine, métabolite monométhylé de l'apomorphine [8], l' amantadine, et les agonistes D2 : lisuride [6], SND 919 (pramipexole), B-HT 920 [41], B-HT 958, CQ 32-084, CQP 201-403 [42], RU 24213 [43], bromocriptine [63], LY 171555 (quinpirole [139]), LY 163502 (quinelorane [33, 111]), 3-PPP et RDS-127. L'injection d'apomorphine, de LY 171555 et de 3-PPP dans le noyau paraventriculaire de l'hypothalamus déclenche également des érections chez le rat [87]. Le noyau paraventriculaire émet des projections ocytocinergiques directes vers les neurones proérectiles de la moelle épinière sacrée. L'administration de naloxone potentialise les effets proérectiles de la N-n-propyl-norapomorphine [40] et de l'apomorphine [13]. L'apomorphine et le LY 171555 augmentent la production de monoxyde

Tableau 1 : Effets proérectiles de l'apomorphine : doses et voies d'injection.

\begin{tabular}{|c|c|c|c|}
\hline dose & Voie d'injection & espèce & références \\
\hline $40-120 \mu \mathrm{g} / \mathrm{kg}$ & sc & rat & $5,87,88$ \\
\hline $5 \mathrm{ng}$ & intra-PVN & Rat & 87 \\
\hline $25-500 \mu \mathrm{g} / \mathrm{kg}$ & ip & rat & 11 \\
\hline $50-600 \mu \mathrm{g} / \mathrm{kg}$ & $\mathrm{SC}, \mathrm{im}$ & singe & 48,110 \\
\hline $9 \mu \mathrm{g} / \mathrm{kg}$ & $\mathrm{se}$ & homme & 29 \\
\hline $0,25-1 \mathrm{mg}$ & sc & homme & $75,76,77$ \\
\hline $2,4-5 \mathrm{mg}$ & sl & Homme+Parkinson & 102 \\
\hline $2-3 \mathrm{mg}$ & sl & homme & $61,98,132,133$ \\
\hline
\end{tabular}


Tableau 2 : Produits agissant sur le contrôle central de l'érection. Les agonistes D2 sont proérectiles, et miment les effets de l'apomorphine. Les antagonistes suppriment les effets proérectiles de l'apomorphine. Ils agissent soit directement sur les circuits dopaminergiques centraux (antagonistes D2), soit sur d'autres systèmes contrôlant les voies dopaminergiques centrales ou sur des étapes en aval de la transmission dopaminergique.

\begin{tabular}{lll}
\hline Agonistes D2 & Antagonistes & Classe des antagonistes \\
\hline Lisuride & Métoclopramide & Antagoniste D2 \\
SND919 & Halopéridol & Id \\
B-HT920 & Sulpiride & Id \\
B-HT958 & Eticlopride & Id \\
CQ 32-084 & Pindolol & Agoniste 5-HT \\
CQP 201-403 & Buspirone & Id \\
RU24213 & 8-OHDPAT & Id \\
Bromocriptine & S-20499 & Id \\
LY171555 & Neurotensine & Neuropeptide \\
LY163502 & Morphine & \\
3-PPP & Muscimol & Agoniste GABA \\
RDS127 & Baclofen & id \\
& Antagonistes OT & \\
\hline
\end{tabular}

d'azote (NO) et de ses métabolites, NO2- et NO3-, dans le noyau paraventriculaire [94]. Chez le rat éveillé, l'effet proérectile de l'apomorphine attesté par des augmentations de la pression intracaverneuse [14] est potentialisé par l'administration de sildénafil [2]. Chez le rat anesthésié, l'injection d'apomorphine par voie intraveineuse, intrathécale ou dans le noyau paraventriculaire déclenche également des augmentations de la pression intracaverneuse $[21,52,53]$.

Les effets proérectiles de l'apomorphine sont abolis après que l'on a traité les animaux par différents produits. Le tableau 2 résume les effets potentialisateurs ou antagonistes de ces produits sur l'érection déclenchée par l'apomorphine. Parmi les produits inhibiteurs on compte : les antagonistes dopaminergiques à action centrale comme la métoclopramide [58], l'halopéridol, le sulpiride et l'éticlopride $[79,104]$, les agonistes du sous-type 5HT1A des récepteurs sérotoninergiques (pindolol, buspirone, 8-OHDPAT, $(+) \mathrm{S}$ 20499 [80, 122], la neurotensine [101], la morphine [13, 95], la scopolamine, antagoniste muscarinique [80], la cystéamine, un produit qui déplète les stocks de somatostatine centrale [83], la cycloheximide, un produit qui inhibe la synthèse des protéines [120], enfin les agonistes des soustypes A (muscimol) et $\mathrm{B}$ (baclofen) des récepteurs GABAergiques [140]. Les effets proérectiles de l'apomorphine sont également abolis après hypophysectomie [121], lésion du noyau paraventriculaire [5], du septum médian, de la fimbria et du fornix $[79,89]$, après injection intracérébroventriculaire d'antagonistes de l'ocytocine [88] et d'in- hibiteurs de la synthèse du monoxyde d'azote $[90,91]$.

On conclut de ces expériences que les effets proérectiles de l'apomorphine recrutent des récepteurs dopaminergiques D2 centraux, en amont d'une cascade de voies polysynaptiques, incluant en particulier des étapes ocytocinergiques et nitrergiques.

RDS-127 et SND 919 déclenchent l'éjaculation chez le rat éveillé $[25,44]$. L'amantadine, le quinelorane et l'apomorphine délivrés par voie systémique, comme le quinelorane et l'apomorphine injectés dans l'aire préoptique médiane, déclenchent l'éjaculation chez le rat éveillé et anesthésié [9, $16,45,46,76,105,124]$. L'apomorphine à la dose de 50 $\mu \mathrm{g} / \mathrm{kg}$ (une dose qui déclenche des érections) abolit l'effet proéjaculateur de la cholécystokinine [106], et l'éticlopride abolit les effets proéjaculateurs de SND 919 [44].

\section{ALTÉRATION DE LA NEUROTRANS- MISSION DOPAMINERGIQUE CENTRALE CHEZ L'HOMME}

La maladie de Parkinson est provoquée par la dégénérescence de la voie dopaminergique nigrostriée. Ceci a pour effet une chute de dopamine dans le striatum [123]. Des précurseurs de la dopamine traversant la barrière hématoencéphalique, par exemple la L-DOPA, ou des agonistes dopaminergiques comme l'apomorphine et la bromocriptine ont été utilisés pour traiter ces malades [118]. Des 
patients traités par la L-DOPA, l'apomorphine ou la bromocriptine décrivent l'apparition d'érections, une meilleure libido ou une augmentation de leur intérêt pour le sexe $[18,19,27,70,102,130]$. Les effets de l'apomorphine chez ces patients doivent être évalués avec prudence [61]. En effet, il existe une chute considérable de la dopamine centrale chez les patients parkinsoniens, par conséquent leur réponse aux agonistes dopaminergiques sont probablement différentes de celles des autres patients.

Les antagonistes de la dopamine déclenchent des signes cliniques proches de la maladie de Parkinson. De tels produits sont utilisés pour le traitement de la schizophrénie. Chez les patients schizophrènes, l'amantadine et l'apomorphine déclenchent des érections, et l'amantadine améliore la libido des patients, mais n'a pas d'effet sur l'éjaculation [131, 137].

Les effets proérectiles de l'apomorphine ont été décrits chez des patients traités pour lutter contre l'alcoolisme chronique [117]. Dans cette étude, $63 \%$ des patients traités par l'apomorphine décrivent des érections provoquées par le traitement.

\section{DYSFONCTIONS ÉRECTILES CHEZ L'HOMME ET MODÈLES ANIMAUX}

L'âge, l'hypertension, le diabète, un faible niveau d'hormones stéroïdes sexuelles, l'hyperprolactinémie, l'hypogonadisme, les lésions de la moelle épinière, les traitements des états dépressifs à l'aide des inhibiteurs de recapture de la sérotonine sont autant de situations au cours desquelles apparaissent les dysfonctions érectiles. Des modèles animaux ont été développés qui miment ces situations, et ils ont été utilisés pour tenter de comprendre les mécanismes et l'origine des dysfonctions [51]. Les effets de l'apomorphine ont été testés dans quelques uns de ces modèles.

\section{Apomorphine et diabète.}

Des patients diabétiques souffrant de dysfonctions érectiles rapportent une amélioration de l'érection lorsqu'ils sont traités avec l'apomorphine [108]. Chez le rat, des résultats contradictoires ont été obtenus. Dans une étude, l'apomorphine ne déclenche pas d'érection chez des rats rendus diabétiques par un traitement avec la streptozotocine [136]. En revanche une autre étude montre qu'une majorité de rats rendus diabétiques par le même traitement présentent des érections en réponse à l'apomorphine [38]. Dans cette même étude, alors que seulement la moitié des rats diabétiques copulent avec une femelle réceptive, l'apomorphine déclenche une activité sexuelle chez certains rats qui n'en ont pas.

\section{Apomorphine et hormones stéroïdes sexuelles.}

La castration induit une chute de l'activité sexuelle [86].
Des rats mâles castrés ne copulent plus, et ne présentent plus d'érection réflexe. Ces animaux ne présentent pas non plus d'érection en réponse à des injections d'apomorphine $[13,57,59,92]$. La castration induit une chute de la quantité de dopamine dans l'hypothalamus [70] et le noyau accumbens chez le rat [97]. Dans l'aire préoptique, la castration induit une chute de la quantité de dopamine extracellulaire, alors qu'elle augmente la quantité de dopamine intracellulaire [35]. La supplémentation des rats mâles castrés par l'oestradiol ou par la testostérone restaure la copulation. Chez les rats castrés et traités par des doses de testostérone restaurant de façon incomplète l'activité sexuelle, l'injection de L-DOPA et de pargyline, un inhibiteur de la monoamine-oxydase, déclenche une agumentation de la libido et de la performance sexuelle $[81,82]$. Le traitement par la testostérone des rats castrés induit une augmentation de la dopamine dans l'hypothalamus, incluant l'aire préoptique médiane $[70,97,112]$. Chez des rats mâles castrés, l'apomorphine délivrée par voie systémique ou par injection dans l'aire préoptique médiane augmente le nombre des montes [116]. Chez le mâle, la testostérone agit sur l'ensemble du névraxe sexuel : on a démontré la présence de récepteurs des androgènes dans les noyaux hypothalamiques contrôlant le comportement sexuel, dans les réseaux de neurones spinaux sacrés contrôlant les organes génitaux, dans les corps cellulaires des neurones des ganglions rachidiens sacrés (afférences sensitives) innervant la région génitale et dans les neurones du plexus sacré (efférences postganglionnaires parasympathiques) innervant le pénis. De plus, les hormones stéroïdes sont d'importantes régulatrices de la transcription, il est par conséquent difficile de leur attribuer un seul tissu cible. L'effet permissif des hormones stéroïdes sexuelles sur le contrôle dopaminergique du comportement sexuel est également présent chez la femelle [69].

La testostérone restaure les érections déclenchées par l'apomorphine, alors que la dihydrotestostérone et l'oestradiol sont inefficaces $[59,92]$. Les souris qui ne synthétisent pas le récepteur alpha des œstrogènes ne copulent pas. Lorsque ces mâles sont traités par l'apomorphine, ils copulent avec des femelles réceptives [134]. Ces données suggèrent que les cibles centrales de la testostérone sont en partie situées en amont des circuits dopaminergiques contrôlant le comportement sexuel.

\section{Apomorphine et hyperprolactinémie.}

La dopamine, libérée par des terminaisons nerveuses dans le système porte hypophysaire, gagne l'hypophyse. Elle inhibe la sécrétion de prolactine en agissant sur la membrane et le génome des cellules cibles [10]. Les produits dopaminergiques sont utilisés dans le traitement de l'hyperprolactinémie. Chez l'homme l'hyperprolactinémie provoque des dysfonctions sexuelle et érectile [20]. On peut greffer des implants d'hypophyse chez le rat. Ces implants ne 
reçoivent plus d'inhibition des voies dopaminergiques, et sécrètent de la prolactine en permanence. L'induction d'une hyperprolactinémie à court terme stimule le comportement sexuel du rat [34]. A long terme, on mesure une diminution des érections réflexes, des montes et des intromissions [31]. Si l'on réalise des injections de prolactine, les érections réflexes ne sont pas touchées [113]. Les effets délétères de la prolactine sur l'érection sont indépendants des effets sur l'excitation sexuelle [32]. Les rats hyperprolactinémiques présentent moins d'érections que des rats témoins en réponse à l'apomorphine [115]. La prolactine agit directement sur le pénis [4] et dans le système nerveux central [113]. L'agoniste dopaminergique RDS-127 diminue les quantités de prolactine d'un facteur $6 \mathrm{chez}$ le rat [23]. Chez des patients hyperprolactinémiques traités par la bromocriptine, agoniste dopaminergique nón sélectif, on mesure une chute des quantités de prolactine sérique, et une amélioration de l'érection. On a rapporté des érections induites par l'apomorphine chez un patient hyperprolactinémique [74]. On améliore la composition du sperme, la fonction gonadique et la sexualité de patients macroprolactinémiques par la bromocriptine ou la cabergoline [30].

\section{Agonistes dopaminergiques indirects et inhibiteurs sélectifs de recapture de la sérotonine}

Les patients souffrant de dépression sont traités par des inhibiteurs sélectifs de recapture de la sérotonine. Ces patients rapportent une diminution du désir et une inhibition de l'orgasme. L'amantadine et la D-amphétamine ont été utilisés chez les patients traités par les inhibiteurs sélectifs de la sérotonine et souffrant de dysfonction sexuelle. Certains parmi les patients ainsi traités décrivent une amélioration de leurs symptômes [7, 49].

\section{RECENTS DEVELOPPEMENTS DANS L'UTILISATION DE L'APOMORPHINE DANS LE TRAITEMENT DES DYSFONC- TIONS ERECTILES CHEZ L'HOMME}

Chez l'homme sain, l'apomorphine déclenche l'érection $[28,29,75]$. Cet effet proérectile apparaît sans aucune autre stimulation, et amplifie les effets proérectiles d'une stimulation sexuelle visuelle [28]. Les essais cliniques utilisant l'apomorphine délivrée par voie sublinguale décrivent un effet proérectile du produit chez des patients souffrant de dysfonctions érectiles $[60,61,76,77,119]$.

L'administration répétée d'apomorphine améliore le succès de la prise du produit $[61,62]$. L'apomorphine sublinguale est prescrite aujourd'hui aux doses de $2 \mathrm{mg}$ et $3 \mathrm{mg}$ (chlorhydrate d'apomorphine, soit respectivement $1,71 \mathrm{mg}$ et $2,56 \mathrm{mg}$ d'apomorphine). Le pic de concentration plasmatique d'apomorphine apparaît 70 minutes après l'adminis- tration sublinguale [98]. La latence de l'érection est inférieure à 20 minutes. Les effets secondaires du produit, décrits par au moins $2 \%$ des patients, sont : nausée, mal de tête, vertige, rhinite, pharyngite [132]. Des études cliniques réalisées en double-aveugle montrent que le pourcentage de patients ayant une érection suffisamment rigide pour un rapport sexuel est plus important dans le groupe traité par l'apomorphine comparé au groupe traité par le placebo. L'un des effets secondaires de l'apomorphine, la nausée, est dose-dépendant, et diminue avec la répétition des prises [36]. Les résultats d'une étude clinique réalisée en Europe confirment la sécurité et la tolérance du traitement par l'apomorphine sublinguale [133]. L'apomorphine n'a pas d'effets sur la libido chez l'homme [98]. Les effets de l'apomorphine sur la pression artérielle ont été étudiés. Chez le chien, les doses d'apomorphine qui provoquent une réaction émétique $(0,05 \mathrm{mg} / \mathrm{kg})$ n'ont pas de propriétés cardiotoxiques [99]. Chez les patients traités par des agents anti-hypertenseurs et des nitrates à courte durée d'action, l'apomorphine sublinguale $(2-6 \mathrm{mg})$ n'a pas plus d'effets cliniques sur la fréquence cardiaque ni sur le pression artérielle que l'apomorphine seule [39]. Des études réalisées chez le chien et le rat révèlent que l'apomorphine n'a pas d'effet sur le poids des organes génitaux, ni sur la mobilité et le nombre des spermatozoïdes, ni sur l'index de fertilité [138]. Chez l'homme les effets proérectiles de la bromocriptine ne sont pas définitivement avérés : le produit est sans effet selon certains auteurs [26], ou bien améliore 1'obtention d'une érection [76, 77]. La L-DOPA augmente de $50 \%$ la fréquence des érections nocturnes et la durée totale des érections pendant le sommeil [64].

\section{CONCLUSIONS}

Les voies dopaminergiques centrales jouent un rôle majeur dans le contrôle du comportement sexuel et de l'érection [54]. L'apomorphine, agoniste dopaminergique à effet central, rejoint les traitements de première intention comme d'autres produits proérectiles délivrés par voie orale, le vacuum et la psychothérapie [132]. En tant que produit à effet central, l'apomorphine peut être comparé au melanotan II, agoniste des récepteurs de la mélanocortine [135]. En tant que produit délivré per os, l'apomorphine rejoint les inhibiteurs des phosphodiestérases [56] et les antagonistes des récepteurs alpha-adrénergiques [55]. L'apparition sur le marché des médicaments de produits permettant une prise orale a considérablement amélioré le confort des patients et l'acceptabilité du traitement.

Il reste cependant à répondre aux questions suivantes :

\section{Les effets de l'apomorphine sont-ils spécifiques d'une seule cible?}


En tant qu'agoniste dopaminergique à effet central, l'apomorphine peut contrôler d'autres viscères. Elle améliore la miction chez les patients parkinsoniens [22] et déclenche des contractions de la vessie chez le rat, un effet partagé avec la L-DOPA [71, 72].

2. Certaines dysfonctions érectiles ont-elles pour origine une altération de la transmission dopaminergique centrale ?

Selon une hypothèse émise par Lal et coll. [76] la dysfonction érectile de certains patients aurait pour origine une telle altération fonctionnelle centrale.

3. L'apomorphine peut-elle être proposée chez la femme?

La bromocriptine déclenche la tumescence du clitoris [17]. Chez l'animal, l'apomorphine améliore l'arrivée de sang dans le clitoris et le vagin [129]. Chez la rate castrée, la quantité de dopamine augmente dans l'hypothalamus en réponse à la supplémentation par l'oestradiol et la progestérone, ou à la stimulation de la région génitale [84]. Ces résultats préliminaires montrent un effet central de la dopamine dans le contrôle du comportement sexuel. En revanche, au moins chez le rat, la dopamine n'a pas d'effet sur la proceptivité ni sur la réceptivité, les deux attitudes comportementales importantes du comportement sexuel de la femelle [93].

\section{RÉFÉRENCES}

1. ANDERSSON K.E. : Pharmacology of penile erection. Pharmacol. Rev., 2001, $53:$ 417-450.

2. ANDERSSON K.E., GEMALMAZ H., WALDECK K., CHAPMAN T.N., TUTTLE J.B., STEERS W.D. : The effect of sildenafil on apomorphine-evoked increases in intracavernous pressure in the awake rat. J. Urol., 1999, $161: 1707-1712$.

3. ANDERSSON K.E, WAGNER G. : Physiology of penile erection. Physiol. Rev., 1995, 75 : 191-236.

4. AOKI H., FUJIOKA T., MATSUZAKA J., KUBO T., NAKAMURA K., YASUDA N. : Suppression by prolactin of the electrically induced erectile response through its direct effect on the corpus cavernosum penis in the dog. J. Urol., 1995, 154:595600 .

5. ARGIOLAS A., MELIS M.R., MAURI A., GESSA G.L. : Paraventricular nucleus lesion prevents yawning and penile erection induced by apomorphine and oxytocin but not by ACTH in rats. Brain Res., 1987 : 421, 349-352.

6. BAGGIO G., FERRARI F. : The role of dopaminergic receptors in the behavioral effects induced by lisuride in male rats. Psychopharmacol., 1983, 80 : 38-42.

7. BALOGH S., HENDRICKS S.E., KANG J. : Treatment of fluoxetine-induced anorgasmia with amantadine. J. Clin. Psychiatry, 1992, 53 : 212-213.

8. BARALDI M., BENASSI-BENELLI A., BERNABEI M.T., CAMERONI R., FERRARI F., FERRARI P. : Apocodeine-induced stereotypies and penile erection in rats. Neuropharmacology, $1979,18: 165-169$.
9. BAZZETT T.J., EATON R.C., THOMPSON J.T., MARKOWSKI V.P., LUMLEY L.A., HULL E.M. : Dose dependent D2 effects on genital reflexes after MPOA injections of quinelorane and apomorphine. Life Sci., 1991, 48 : 2309-2315.

10. BEN-JONATHAN N., HNASKO R. : Dopamine as a prolactin (PRL) inhibitor. Endocrine Rev., 2001, 22 : 724-763.

11. BENASSI-BENELLI A., FERRARI F., PELLEGRINI-QUARANTOTTI B. : Penile erection induced by apomorphine and Nn-propyl-norapomorphine in rats. Arch. Int. Pharmacodyn. Ther., 1979, 242: 241-247.

12. BENASSI-BENELLI A., FERRARI F. : Activation with N-npropyl-norapomorphine (NPA) of the male rat copulatory behavior. Experientia, 1979, $35:$ 645-646.

13. BERENDSEN H.H.G., GOWER A.J. : Opiate-androgen interactions in drug-induced yawning and penile erections in the rat. Neuroendocrinology, 1986, $42: 185-190$.

14. BERNABE J., RAMPIN O., SACHS B.D., GIULIANO F. : Intracavernous pressure during erection in rats : an integrative approach based on telemetric recording. Am. J. Physiol., 1999, $276: 441-449$.

15. BITRAN D., HULL E.M. : Pharmacological analysis of male sexual behavior. Neurosci. Biobehav. Rev., 1987, 11 : 365-389.

16. BITRAN D., THOMPSON J.T., HULL E.M., SACHS B.D. : Quinelorane (LY 163502), a D2 dopamine receptor agonist, facilitates seminal emission, but inhibits penile erection in the rat. Pharmacol. Biochem. Behav., 1989, 34 : 453-458.

17. BLIN O., SCHWERTSCHLAG U.S., SERRATRICE G. : Painful clitoral tumescence during bromocriptine therapy. Lancet, 1991, $337: 1231-1232$.

18. BOWERS M.B., VAN WOERT M., DAVIS L. : Sexual behavior during L-DOPA treatment for Parkinsonism. Am. J. Psychiatry, 1971, $127: 1691-1693$.

19. BROWN E., BROWN G.M., KOFMAN O., QUARRINGTON B. : Sexual function and affect in Parkinsonian men treated with L-DOPA. Am. J. Psychiatry, 1978, 135 : 1552-1555.

20. BUVAT J., LEMAIRE A., BUVAT-HERBAUT M., FOURLINNIE J.C., RACADOT A., FOSSATI P. : Hyperprolactinemia and sexual function in men. Horm. Res., 1985, 22, 196-203.

21. CHEN K.K., CHAN J.Y., CHANG L.S. : Dopaminergic neurotransmission at the paraventricular nucleus of the hypothalamus in central regulation of penile erection in the rat. J. Urol., 1999, $162: 237-242$.

22. CHRISTMAS T.J., KEMPSTER P.A., CHAPPLE C.R. et al. : Role of subcutaneous apomorphine in parkinsonian voiding dysfunction. Lancet, 1988, 2 : 1451-1453.

23. CLARK J.T., SMITH E.R., STEFANIK M.L., ARNERIC S.P., LONG J.P., DAVIDSON J.M. : Effects of a novel dopaminereceptor agonist, RDS-127 (2-N,N-di-n-propylamino-4,7-dimethoxyindane), on hormone levels and sexual behavior in the male rat. Physiol. Behav., 1982, 29 : 1-6.

24. CLARK J.T., STEFANIK M.L., SMTTH E.R., DAVIDSON J.M.: Further studies on alterations in male rat copulatory behavior induced by the dopamine-receptor agonist RDS- 127 . Pharmacol. Biochem. Behav., 1983, 19 : 781-786.

25. CLARK J.T., PEROUTKA S.J., CIARANELLO R.D., SMITH E.R., DAVIDSON J.M. : Central effects of RDS-127 : sexual behavior after intracerebroventricular administration and in vitro receptor binding studies. Behav. Brain Res., 1985, 18 : 251-260. 
26. COOPER A.J. : Bromocriptine in impotence. Lancet, 1977, 2 : $8037,567$.

27. COURTY E., DURIF F., ZENUT M., COURTY P., LAVARENNE J. : Psychiatric and sexual disorders induced by apomorphine in Parkinson's disease. Clin. Neuropharmacol., 1997, 20 : 140-147.

28. DANJOU P., LACOMBLEZ L., WAROT D., PUECH A.J. : Assessment of erectogenic drugs by numeric plethysmography, J. Pharm. Meth., 1989, $21:$ 61-69.

29. DANJOU P., ALEXANDRE L., WAROT D., LACOMBLEZ L., PUECH A.J. : Assessment of erectogenic properties of apomorphine and yohimbine in man. Brit. J. Clin. Pharmacol., 1988, 26: 733-739.

30. DE ROSA M., COLAO A., DI SARNO A. et al. :Treatment rapidly improves gonadal function in hyperprolactinemic males: a comparison with bromocriptine. Eur. J. Endoccrinol., 1998, $138: 286-293$.

31. DOHERTY P.C., BAUM M.J., TODD R.B. : Effects of chronic hyperprolactinemia on sexual arousal and erectile function in male rats. Neuroendocrinology, 1986, 42 : 368-375.

32. DOHERTY P.C., WU DE, MATT K.S. : Hyperprolactinemia preferentially inhibits erectile function in adrenalectomized male rats. Life Sci., 1990, 47 : 141-148.

33. DOHERTY P.C., WISLER P.A. : Stimulatory effects of quinelorane on yawning and penile erection in the rat. Life Sci., 1994, $54: 507-514$.

34. DRAGO F., PELLEGRINI-QUARANTOTTI B., SCAPAGNINI U., GESSA G.L. : Short-term endogenous hyperprolactinemia and sexual behavior of male rats. Physiol. Behav., 1981, 26 : 277-279.

35. DU J., LORRAIN D.S., HULL E.M. : Castration decreases extracellular, but increases intracellular, dopamine in medial preoptic area of male rats. Brain Res., 1998, 782, 11-17.

36. DULA E., KEATING W., SIAMI P.F., EMDONDS A., O'NEIL, J., BITTLER S., and the APO Study Group : Efficacy and safety of fixed-dose and dose-optimization regimens of sublingual apomorphine versus placebo in men with erectile dysfunction. Urology, 2000, $56: 130-135$.

37. EATON R.C., MARKOWSKI V.P., LUMLEY L.A., THOMSON J.T., MOSES J., HULL E.M. : D2 receptors in the paraventricular nucleus regulate genital responses and copulation in male rats. Pharmacol. Biochem. Behav., 1991, 39 177-181.

38. ELABBADY A., HASSOUNA M.M., ELHILALI M. : Apomorphine versus mating behavior in testing erectile capabilities of diabetic rats. Urology, 1995, $45:$ 715-719.

39. FAGAN T.C., BUTTLER S., MARBURY T., TAYLOR A., EDMONDS A. and the SL Apo Study Group : Cardiovascular safety of sublingual apomorphine in patients on stable doses of oral antihypertensive agents and nitrates. Am. J. Cardiol., 2001, $88: 760-766$.

40. FERRARI F., BAGGIO G. : Potentiation of the aphrodisiac effect of $\mathrm{N}$-n-propyl-norapomorphine by naloxone. Eur. J. Pharmacol., 1982, 81 : 321-326.

41. FERRARI F. : Sexual excitement and stretching and yawning induced by B-HT 920. Pharmacol. Res. Comm., 1985, 17 : 557564.

42. FERRARI F, GIULIANI D. : Behavioural effects induced in rats and chicks by D2 dopamine agonists. Physiol. Behav., 1993, 54, 695-700.
43. FERRARI F., PELLONI GIULIANI D. : Behavioural evidence that different neurochemical mechanisms underly stretchingyawning and penile erection induced in male rats by SND 919, a new selective D2 dopamine receptor agonist. Psychopharmacology, 1993, 113: 172-176.

44. FERRARI F., GIULIANI D. : The selective D2 dopamine receptor antagonist eticlopride counteracts the ejaculatio praecox induced by the selective D2 dopamine agonist SND 919 in the rat. Life Sci., 1994, 55 : 1155-1162.

45. FERRAZ M.R., SANTOS R. : Amantadine stimulates sexual behavior in male rats. Pharmacol. Biochem. Behav., 1995, 51 : 709-714.

46. FERRAZ M.R., FERRAZ M.M., SANTOS R. : How REM sleep deprivation and amantadine affect male rat sexual behavior. Pharmacol. Biochem. Behav., 2001, 69 : 325-332.

47. FUMERO B., FERNANDEZ-VERA J.R., GONZALEZ-MORA J.L., MAS M. : Changes in monoamine turnover in forebrain areas associated with masculine sexual behavior : a microdialysis study. Brain Res., 1994, 662 : 233-239.

48. GISOLFI C.V., MOAR F., WALL P.T. : Dopamine and temperature regulation in the primate : effects of apomorphine and pimozide. Brain Res. Bull., 1980, 5 : 349-352.

49. GITLIN M.J. : Effects of depression and antidepressants on sexual functioning. Bull. Menninger. Clin., 1995, 59 : 232-248.

50. GIULIANI D., FERRARI F. : Differential behavioral response to dopamine D2 agonists by sexually naïve, sexually active, and sexually inactive male rats. Behav. Neurosci., 1996, 110 : 802808.

51. GIULIANO F., RAMPIN O., McKENNA K. : Animal models used in the study of erectile dysfunction. In : CC Carson III, RS Kirby, I Goldstein eds. Textbook of erectile dysfunction, Oxford, ISIS Medical Media, 1999 : chapt. 5, 43-49.

52. GIULIANO F., ALLARD J., RAMPIN O. et al. : Spinal proerectile effect of apomorphine in the anesthetized rat. Int. J. Impotence Res., 2001, 13 : 110-115.

53. GIULIANO F., ALLARD J., RAMPIN O. et al. : Pro-erectile effect of systemic apomorphine : existence of a spinal site of action. J. Urol., 2002, $167: 402-406$.

54. GIULIANO F., ALLARD J. : Dopamine and male sexual function. Eur. Urol., 2001, 40 : 601-608.

55. GOLDSTEIN I., CARSON C., ROSEN R., ISLAM A. : Vasomax for the treatment of male erectile dysfunction. World $\mathrm{J}$. Urol., 2001, 19 : 51-56.

56. GOLDSTEIN I., LUE T.F., PADMA-NATHAN H., ROSEN R.C., STEERS W.D., WICKER P.A. : Oral sildenafil in the treatment of erectile dysfunction. New Engl. J. Med., 1998, 338 : 1397-1404.

57. HEATON J.P.W., VARRIN S.J., MORALES A. : The characterization of a bio-assay of erectile function in a rat model. J. Urol., 1991, $145: 1099-1102$.

58. HEATON J.P.W., VARRIN S.J. : Metoclopramide decreases apomorphine-induced yawning and penile erection. Pharmacol. Biochem. Behav., 1991, 38 : 917-920.

59. HEATON J.P.W., VARRIN S.J. : Effects of castration and exogenous testosterone supplementation in an animal model of penile erection. J. Urol., 1994, $151:$ 797-800.

60. HEATON J.P.W., MORALES A., ADAMS M.A., JOHNSTON B., EL-RASHIDY R. : Recovery of erectile function by the oral administration of apomorphine. Urol., 1995, 45 : 200-206. 
61. HEATON J.P.W. : Key issues from the clinical trials of apomorphine SL. World J. Urol., 2001, 19 : 25-31.

62. HEATON J.P., DEAN J., SLEEP D.J. : Sequential administration enhances the effect of apomorphine SL in men with erectile dysfunction. Int. J. Impot. Res., 2002, 14 : 61-64.

63. HOLMGREN B., URBA-HOLMGREN R., TRUCIOS N., ZERMENO M., EGUIBAR J.R. : Association of spontaneous and dopaminergic-induced yawning and penile erections in the rat. Pharmacol. Biochem. Behav., 1985, 22 : 31-35.

64. HORITA H., SATO Y., ADACHI H. et al. : T. Effects of levodopa on nocturnal penile tumescence: a preliminary study. J. Androl., 1998, 19 : 619-624.

65. HULL E.M., BITRAN D., PEHEK E.A., WARNER R.K., BAND L.C., HOLMES G.M. : Dopaminergic control of male sex behavior in rats: effects of an intracerebrally-infused agonist. Brain Res., 1986, 370 : 73-81.

66. HULL E.M., BAZZETT T.J., WARNER R.K., EATON R.C, THOMPSON J.T. : Dopamine receptors in the ventral tegmental area modulate male sexual behavior in rats. Brain Res., 1990, 512:1-6.

67. HULL E.M., EATON R.C., MARKOWSKI V.P., MOSES J., LUMLEY L.A., JOUCKS J.A. : Opposite influence of medial preoptic D1 and D2 receptors on genital reflexes. Implication for copulation. Life Sci., 1992, 51 : 1705-1713.

68. HULL E.M., DU J., LORRAIN D.S., MATUSZEWICH L. : Extracellular dopamine in the medial preoptic area : implications for sexual motivation and hormonal control of copulation. $J$. Neurosci., 1995, 15 : 7465-7471.

69. HULL E.M., LORRAIN D.S., DU J. et al. : Hormone-neurotransmitter interactions in the control of sexual behavior. Behav. Brain Res., 1999, 105 : 105-116.

70. HYYPPA M., RINNE U.K., SONNINEN V. : The activating effect of L-DOPA treatment on sexual functions and its experimental background. Acta Neurol. Scand. Suppl., 1970, 43 : 223224.

71. ISHIZUKA O., MATTIASSON A., STEERS W.D., ANDERSSON K.E. : Effects of spinal alpha-1 adrenoceptor antagonism on bladder activity induced by apomorphine in conscious rats with and without bladder outlet obstruction. Neurourol. Urodyn., 1997, $16: 191-200$.

72. ISHIZUKA O., PANDITA R.K., MATTIASSON A., STEERS W.D., ANDERSSON K.E. : Stimulation of bladder activity by volume, L-DOPA and capsaicin in normal conscious rats effects of spinal alpha-1 adrenoceptor blockade. Naunyn Schmiedebergs Arch. Pharmacol., 1997, 335 : 787-793.

73. KAPLAN J.M., HAO J., SODERSTEN P. : Apomorphine induces ejaculation in chronic decerebrate rats. Neurosci. Lett., 1991, $129:$ : 205-208.

74. KIELY M.E., THAVUNDAYIL J.X., LAL S. : Effect of blood sampling on apomorphine-induced penile tumescence in erectile impotence : a case report. J. Psychiatry Neurosci., $20: 233-235$.

75. LAL. S., ACKMAN D., THAVUNDAYIL J.X., KIELY M.E., ETIENNE P. : Effect of apomorphine, a dopamine receptor agonist, on penile tumescence in normal subjects. Prog. Neuropsychopharmacol. Biol. Psychiatry, 1984, 8 : 695-699.

76. LAL S., LARYEA E., THAVUNDAYIL I.X. : Apomorphineinduced penile tumescence in impotent patients - preliminary findings. Prog. Neuro-psychopharmacol. Biol. Psychiatry, 1987, $11: 235-242$.
77. LAL S., TESFAYE Y., THAVUNDAYIL J.X. et al. : Apomorphine : clinical studies on erectile impotence and yawning. Prog. Neuropsychopharmacol. Biol. Psychiatry, 1989, 13 : 329-339.

78. LAL S., KIELY M.E., THAVUNDAYIL J.X., STEWART J.D., ASSALIAN P., ACKMAN C.F. : Effect of bromocriptine in patients with apomorphine-responsive erectile impotence : an open study. J. Psychiatry Neurosci., 1991, $16: 262-266$.

79. MAEDA N., MATSUOKA N., YAMAGUCHI I. : Septohippocampal cholinergic pathway and penile erections induced by dopaminergic and cholinergic stimulants. Brain Res., $1990,537: 163-168$.

80. MAEDA N., MATSUOKA N., YAMAGUCHI I. : Role of the dopaminergic, serotonergic and cholinergic link in the expression of penile erection in rats. Jpn J. Pharmacol, 1994, 66 : 5966.

81. MALMNAS C.O. : Effects of LSD-25, clonidine and apomorphine on copulatory behavior in the male rat. Acta Physiol. Scand. Suppl., 1973, 395 : 96-128.

82. MALMNAS C.O. : Monoamine precursors and copulatory behavior in the male rat. Acta Physiol. Scand. Suppl., 1973, $395: 47$ 68.

83. MATSUOKA N., MAEDA N., YAMAZAKI M., YAMAGUCHI I. : Brain somatostatin depletion by cysteamine attenuates the penile erection induced by serotonergic and dopaminergic, but not by cholinergic, activation. Brain Res., 1996, 729 : 132-136.

84. MATUSZEWICH L., LORRAIN D.S., HULL E.M. : Dopamine release in the medial preoptic area of female rats in response to hormonal manipulation and sexual activity. Behav. Neurosci., 2000, $114: 772-782$.

85. McKENNA K.E. Neural circuitry involved in sexual function. J. Spinal. Cord. Med, 2001, 24 : 148-154.

86. MEISEL R.L., SACHS B.D. : The physiology of male sexual behavior. In : Knobil E., Neill J.D. eds. The Physiology of Reproduction. New York, Raven Press, 1994, volume 2 : 3-106.

87. MELIS M.R., ARGIOLAS A., GESSA G.L. : Apomorphineinduced penile erection and yawning : site of action in the brain. Brain Res., 1987, 415 : 98-104.

88. MELIS M.R., ARGIOLAS A., GESSA G.L. : Evidence that apomorphine induces penile erection and yawning by releasing oxytocin in the central nervous system. Eur. J. Pharmacol., 1989, $164: 565-570$.

89. MELIS M.R., STANCAMPIANO R., ARGIOLAS A., : Hippocampal oxytocin mediates apomorphine-induced penile erection and yawning. Pharmacol. Biochem. Behav., 1992, 42 : 61-66.

90. MELIS M.R., ARGIOLAS A. : Nitric oxide synthase inhibitors prevent apomorphine- and oxytocin-induced penile erection and yawning in male rats. Brain Res. Bull., 1993, 32 : 71-74.

91. MELIS M.R., STANCAMPIANO R., ARGIOLAS A., : Prevention by Ng-nitro-L-arginine methyl ester of apomorphineand oxytocin-induced penile erection and yawning : site of action in the brain. Pharmacol. Biochem. Behav., 1994, 48 : 799804.

92. MELIS M.R., MAURI A., ARGIOLAS A., : Apomorphine- and oxytocin-induced penile erection and yawning in intact and castrated male rats : effect of sexual steroids. Neuroendocrinology, $1994,59: 349-354$.

93. MELIS M.R., ARGIOLAS A. : Dopamine and sexual behavior. Neurosci. Biobehav. Rev., 1995, 19: 19-38. 
94. MELIS M.R., SUCCU S., ARGIOLAS A. : Dopamine agonists increase nitric oxide production in the paraventricular nucleus of the hypothalamus : correlation with penile erection and yawning. Eur. J. Neurosci., 1996, 8 : 2056-2063.

95. MELIS M.R., SUCCU S, IANNUCCI U,, ARGIOLAS A. : Prevention by morphine of apomorphine- and oxytocin-induced penile erection and yawning : involvement of nitric oxide. Naunyn-Schmiedebergs Arch. Pharmacol., 1997, 335 : 595-600.

96. MISSALE C., NASH S.R., ROBINSON S.W., JABER M., CARON M.G. : Dopamine receptors : from structure to function. Physiol. Rev., 1998, 78 : 189-225.

97. MITCHELL J.B., STEWART J. : Effects of castration, steroid replacement, and sexual experience on mesolimbic dopamine and sexual behaviors in the male rat. Brain Res., 1989, 491 : 116127.

98. MULHALL J.P, : Sublingual apomorphine for the treatment of erectile dysfunction. Expert. Opin. Investig. Drugs, 2002, 11 : 295-302.

99. NAKAYAMA H., NAKAYAMA T., CARNES C.A., STRAUCH S.M., HAMLIN R.L. : Electrophysiologic and hemodynamic effects of apomorphine in dogs. Toxicol. Appl. Pharmacol., 2001, $177: 157-161$.

100. NAPOLI-FARRIS L., FRATTA W., GESSA G.L. : Stimulation of dopamine autoreceptors elicits "premature ejaculation" in rats. Pharmacol. Biochem. Behav., 1984, $20: 69-72$.

101. NOUEL D., COSTENTIN J. : Inhibition of apomorphine-induced yawning and penile erection by neurotensin. Peptides, 1991, $12: 755-759$.

102. O'SULLIVAN J.D., HUGHES A.J. : Apomorphine-induced penile erections in Parkinson's disease. Mov. Disorders, 1998, 13: 536-539.

103. PAGLIETTI E., PELLEGRINI QUARANTOTTI B, MEREU G., GESSA G.L. : Apomorphine and L-DOPA lower ejaculation threshold in the male rat. Physiol. Behav., 1978, 20 559-562.

104. PEHEK E.A., THOMPSON J.T., EATON R.C., BAZZETT T.J., HULL E.M. : Apomorphine and haloperidol, but not domperidone, affect penile reflexes in rats. Pharmacol. Biochem. Behav., 1988, $31: 201-208$.

105. PEHEK E.A., THOMPSON J.T., HULL E.M. : The effects of intracranial administration of the dopamine agonist apomorphine on penile reflexes and seminal emission in the rat. Brain Res., $1989,500: 325-332$.

106. PFAUS J.G., PHILLIPS A.G. : Cholecystokinin facilitates ejaculation in male rats : blockade with proglumide and apomorphine. Eur. J. Pharmacol., 1987, 141 : 331-338.

107. PFAUS J.G., DAMSMA G., NOMIKOS G.G. et al. : Sexual behavior enhances central dopamine transmission in the male rat. Brain Res., 1990, $530: 345-348$.

108. PIERINI A.A., NUSIMOVICH B. : Male diabetic impotence : effects of dopaminergic agents. Arch. Androl., 1981, 6 : 347350.

109. PLEIM E.T., MATOCHIK J.A., BARFIELD R.J., AUERBACH S.B. : Correlation of dopamine release in the nucleus accumbens with masculine sexual behavior in rats. Brain Res., 1990, 524 : 160-163.

110. POMERANTZ S.M. : Apomorphine facilitates male sexual behavior of rhesus monkeys. Pharmacol. Biochem. Behav., $1990,35: 659-664$.
111. POMERANTZ S.M. : Quinelorane (LY 163502), a D2 dopamine receptor agonist, acts centrally to facilitate penile erections of male rhesus monkeys. Pharmacol. Biochem. Behav., 1991, 39 : 123-128.

112. PUTMAN S.K., DU J., SATO S., HULL E.M. : Testosterone restoration of copulatory behavior correlates with medial preoptic dopamine release in castrated male rats. Horm. Behav., 2001, 39: 216-224.

113. REHMAN J., CHRIST G., ALYSKEWYCZ M., KERR E., MELMAN A. : Experimental hyperprolactinemia in a rat model: alteration in centrally mediated neuroerectile mechanisms. Int. J. Impot. Res., 2000, $12: 23-32$.

114. SACHS B.D. : Contextual approaches to the physiology and classification of erectile function, erectile dysfunction, and sexual arousal. Neurosci. Biobehav. Rev., 2000, 24 : 541-560.

115. SATO F., AOKI H., NAKAMURA K., TAGUCHI M., AOKI T., YASUDA N. : Suppressive effects of chronic hyperprolactinemia on penile erection and yawning following administration of apomorphine to pituitary-transplanted rats. J. Androl., 1997, 18: 21-25.

116. SCALETTA L.L., HULL E.M. : Systemic or intracranial apomorphine increases copulation in long-term castrated male rats. Pharmacol. Biochem. Behav., 1990, 37 : 471-475.

117. SCHLATTER E.K.E., LAL S. : Treatment of alcoholism with Dent's oral apomorphine method. Quart. J. Stud. Alc., 1972, 33: 430-436.

118. SCHWAB R.S., AMADOR L.F., LETTVIN J.Y. : Apomorphine in Parkinson's disease. Trans. Am. Neurol. Assoc., 1951, 76 : 251-253.

119. SEGRAVES R.T., BARI M., SEGRAVES K., SPIRNAK P. : Effect of apomorphine on penile tumescence in men with psychogenic impotence. J. Urol., 1991, 145 : 1174-1175.

120. SERRA G., FRATTA W., COLLU M., NAPOLI-FARRIS L., GESSA G.L. : Cycloheximide prevents apomorphine-induced yawning, penile erection and genital grooming in rats. Eur. J. Pharmacol., 1983, 86 : 279-282.

121. SERRA G., COLLU M., LODDO S., CELASCO G., GESSA G.L. : Hypophysectomy prevents yawning and penile erection but not hypomotility induced by apomorphine. Pharmacol. Biochem. Behav, 1983, 19 : 917-919.

122. SIMON P., GUARDIOLA B., BIZOT-ESPIARD J., SCHIAVI P., COSTENTIN J. : 5-HT1A receptor agonists prevent in rats the yawning and penile erections induced by direct dopamine agonists. Psychopharmacology, 1992, $108:$ 47-50.

123. SOURKERS T.L. : Disorders of the basal ganglia. In : Qiegel G., Agranoff B., Albers R.W., Molinoff P. eds. Basic Neurochemistry, $4^{\text {th }}$ edition. New-York, Raven Press, 1989 : 811-825.

124. STEFANICK M.L., SMITH E.R., CLARK J.T., DAVIDSON J.M. : Effects of a potent dopamine receptor agonist, RDS-127, on penile reflexes and seminal emission in intact and spinally transected rats. Physiol. Behav., 1982, $29: 973-978$.

125. SZCZYPKA M.S., ZHOU Q.Y., PALMITER R.D. : Dopaminestimulated sexual behavior is testosterone dependent in mice. Behav. Neurosci., 1998, $112:$ 1229-1235.

126. TAGLIAMONTE A., FRATTA W., DELFIACCO M., GESSA G.L. : Evidence that brain dopamine stimulates copulatory behavior in male rats. Riv. Farm. Therap., 1973, 4 : 177-181. 
127. TAGLIAMONTE A., FRATTA W., GESSA G.L. : Aphrodisiac effect of L-DOPA and apomorphine in male sexually sluggish rats. Experientia, 1974, $30: 381-382$.

128. TAGLIAMONTE A., FRATTA W., DELFIACCO M., GESSA G.L. : Possible stimulatory role of brain dopamine in the copulatory behavior of male rats. Pharmacol. Biochem. Behav., 1974, $2: 257-260$.

129. TARCAN T., SIROKY M.B., PARK K., GOLDSTEIN I., AZADZOI K.M. : Systemic administration of apomorphine improves the hemodynamic mechanism of clitoral and vaginal engorgement in the rabbit. Int. J. Impotence Res., 2000, 12 : 235-240.

130. UITTI R.J., TANNER C.M., RAJPUT A.H., GOETZ C.G., KLAWANS H.L., THIESSEN B, : Hypersexuality with antiparkinsonian therapy. Clin. Neuropharmacol., 1989, 12 : 375-383.

131. VALEVSKI A., MODAI I., ZBARSKI E., ZEMISHLANY Z., WEIZMAN A. : Effect of amantadine on sexual dysfunction in neuroleptic-treated male schizophrenic patients. Clin Neuropharmacol., 1998, 21 : 355-357.

132. VON KEITZ A. : The management of erectile dysfunction in the community. Int. J. Impotence Res., 2001, 13 Suppl 3 : 45-51.

133. VON KEITZ A.T., STROBERG P., BUKOFZER S., MALLARD N., HIBBERD M. : A European multicentre study to evaluate the tolerability of apomorphine sublingual administered in a forced dose-escalation regimen in patients with erectile dysfunction. B.J.U. Int., 2002, 89 : 409-415.

134. WERSINGER S.R., RISSMAN E.F. : Dopamine activates masculine sexual behavior independent of the estrogen receptor alpha. J. Neurosci., 2000, $20:$ 4248-4254.

135. WESSELLS H., FUCIARELLI K., HANSEN J. et al. : Synthetic melanotropic peptide initiates erection in men with psychogenic erectile dysfunction : double-blind, placebo controlled crossover study. J. Urol., 1998, $160: 389-393$.

136. YAMAGUCHI Y., KOBAYASHI H. : Effects of apomorphine, physostigmine and vasoactive intestinal polypeptide on penile erection and yawning in diabetic rats. Eur. J. Pharmacol., 1994, $254: 91-96$.

137. YARYURA-TOBIAS J.A., DIAMOND B., MERLIS S. : The action of L-DOPA on schizophrenic patients; a preliminary report. Curr. Ther. Res., 1970, 12 : 528-531.

138. YOUSSEF A.F, FORT F.L., RONSEN B., SCHROEDER R., WILLIAMS K., AULETTA C. : Evaluation of apomorphine $\mathrm{HCl}$ effects on reproductive endpoints : studies in male rats and dogs. Toxicol. Sci., 1999, 51 : 273-279.

139. ZARRINDAST M.R., SHOKRAVI S., SAMINI M. : Opposite influence of dopaminergic receptor subtypes on penile erection. Gen. Pharmacol., 1992, 23 : 671-675.

140. ZARRINDAST M.R., FARAHVASH H. : Effects of GABAergic drugs on penile erection induced by apomorphine in rats. Psychopharmacol., 1994, 115 : 249-253.

\section{ABSTRACT}

Apomorphine, central dopaminergic pathways and control of penile erection.

\section{Olivier RAMPIN}

Relaxation of penile erectile tissue and increased blood flow in the penile arteries are the two basic local mechanisms of erection. Relaxation is elicited by several agents released by the nerve terminals of sacral parasympathetic pathways (nitric oxide [NO] and vasoactive intestinal polypeptide [VIP]) and endothelial cells. The increased activity of sacral parasympathetic pathways leads to erection. Other molecules (e.g. noradrenaline) released by the nerve endings of sympathetic pathways contract the penile tissue and arteries. A decrease in sympathetic pathway activity can therefore lead to erection. A better understanding of the local mechanisms of penile erection has led to the production of compounds designed to treat erectile dysfunction via a peripheral target. Such compounds are recognised as initiators if they elicit erection per se or as conditioners if they potentiate a mechanism already present.

The central control of penile erection plays an important role in the optimal functioning of the erectile process. Sympathetic and parasympathetic nerves to the penis originate in the spinal cord. In a sexually relevant context, it is likely that a shift of the balance between sympathetic and parasympathetic activities causes erection. This shift is controlled at the spinal cord level by information from the periphery (reflex pathways) and from supraspinal nuclei. Recent experiments have focused on supraspinal nuclei present in the brainstem, pons, and hypothalamus that directly project onto the sacral spinal cord. Pharmacological approaches have revealed an important role for central dopamine in the control of sexual behavior and the genital tract in males. Central dopamine can therefore regulate both sympathetic and parasympathetic pathways. Levels of DA and its metabolites increase in several brain structures during sexual activity. DA agonists, e.g. the $D_{1} / D_{2}$ agonist apomorphine, affect the sexual behavior, erection and ejaculation in a variety of animal species and in humans. Recent clinical investigations have revealed the benefits of the use of apomorphine in patients suffering from erectile dysfunction. Compounds acting centrally, such as apomorphine, can contribute to reorganize the activity of sympathetic and parasympathetic outflows leading to an appropriate recruitment of the autonomic pathways to the genital tract.

Key words : apomorphine, dopamine, erection, ejaculation, erectile dysfunction, sexual behavior. 